\title{
$p$-Minkowski inequalities for mixed intersection bodies
}

\author{
Zhao Chang-jian and Mihály Bencze
}




\title{
p-MINKOWSKI INEQUALITIES FOR MIXED INTERSECTION BODIES
}

\author{
ZHAO CHANG-JIAN AND MIHÁLY BENCZE \\ Received 10 June, 2009
}

\begin{abstract}
In this paper, we establish $p$-Minkowski inequality for mixed intersection bodies, which is a general form of the Minkowski inequality for mixed intersection bodies.
\end{abstract}

2000 Mathematics Subject Classification: 52A40

Keywords: dual mixed volumes, mixed intersection bodies

\section{INTRODUCTION}

One might say the history of intersection bodies began with the paper [4] of Busemann. Intersection bodies were first explicitly defined and named by Lutwak[13]. It was here that the duality between intersection bodies and projection bodies was first made clear. Despite the considerable ingenuity of earlier attacks on the BusemannPetty problem, it seems fair to say that the work [13] of Lutwak represents the beginning of its eventual solution. In [13], Lutwak also showed that if a convex body is sufficiently smooth and not an intersection body, then there exists a centered star body such that the conditions of Busemann-Petty problem holds, but the in the result the inequality's direction is reversed. Following Lutwak, the intersection body of order $i$ of a star body is introduced by Zhang[21]. It follows from this definition that every intersection body of order $i$ of a star body is an intersection body of a star body, and vice versa. As Zhang observes, the new definition of intersection body allows a more appealing formulation, namely: The Busemann-Petty problem has a positive answer in $n$-dimensional Euclidean space if and only if each centered convex body is an intersection body. The intersection body plays an essential role in Busemann's theory [5] of area in Minkowski spaces. The intersection body also plays an important role in the Brunn-Minkowski theory.

In recent years some author including Ball[1,2], Bourgain[3], Gardner[6-8], Schneider[19] and Lutwak[11,12,14-18], et al. have given considerable attention to the Brunn-Minkowski theory and their various generalizations. The purpose of

Research is supported by Zhejiang Provincial Natural Science Foundation of China(Y605065), Foundation of the Education Department of Zhejiang Province of China(20050392). 
this paper is firstly to establish the Minkowski inequality for the dual Quermassintegral sum, which is a generalization of the Minkowski inequality for mixed intersection bodies. Then, the Brunn-Minkowski inequality and the Aleksandrov-Fenchel inequality for mixed intersection bodies are proved and some related results are also given. In this work we shall derive, for intersection bodies, all the analogous inequalities for Lutwak's mixed projection body inequalities [17]. Thus, this work may be seen as additional evidence for the natural duality between intersection and projection bodies.

\section{Notation AND PRELIMinaries}

The setting of this paper is the $n$-dimensional Euclidean space $\mathbb{R}^{n}(n>2)$. Let $\mathbb{C}^{n}$ denote the set of non-empty convex figures (compact, convex subsets) and let $\mathcal{K}^{n}$ denote the subset of $\mathbb{C}^{n}$ consisting of all convex bodies (compact, convex subsets with non-empty interiors) in $\mathbb{R}^{\mathrm{m}}$. We reserve the letter $u$ for unit vectors, and the letter $B$ is reserved for the unit ball centered at the origin. The surface of $B$ is $S^{n-1}$. For $u \in S^{n-1}$, let $E_{u}$ denote the hyperplane, through the origin, that is orthogonal to $u$. We will use $K^{u}$ to denote the image of $K$ under an orthogonal projection onto the hyperplane $E_{u}$. We use $V(K)$ for the $n$-dimensional volume of the convex body $K$. The support function of $K \in \mathcal{K}^{n}, h(K, \cdot)$, is defined on $\mathbb{R}^{n}$ by $h(K, \cdot)=$ $\operatorname{Max}\{x \cdot y: y \in K\}$. Let $\delta$ denote the Hausdorff metric on $\mathcal{K}^{n}$; i.e., for $K, L \in \mathcal{K}^{n}$, $\delta(K, L)=\left|h_{K}-h_{L}\right|_{\infty}$, where $|\cdot|_{\infty}$ denotes the sup-norm on the space of continuous functions, $C\left(S^{n-1}\right)$.

Associated with a compact subset $K$ of $\mathbb{R}^{n}$, which is star-shaped with respect to the origin, its radial function $\rho(K, \cdot): S^{n-1} \rightarrow \mathbb{R}$, is defined (for $u \in S^{n-1}$ ) by $\rho(K, u)=\operatorname{Max}\{\lambda \geq 0: \lambda u \in K\}$. If $\rho(K, \cdot)$ is positive and continuous, $K$ will be called a star body. Let $\varphi^{n}$ denote the set of star bodies in $\mathbb{R}^{n}$.

\section{DuAl MiXed VOLUMeS}

If $K_{1}, \ldots, K_{r} \in \varphi^{n}$ and $\lambda_{1}, \ldots, \lambda_{r} \in \mathbb{R}$, then the radial Minkowski linear combination, $\lambda_{1} K_{1} \tilde{+} \cdots \tilde{+} \lambda_{r} K_{r}$, is defined by

$$
\lambda_{1} K_{1} \tilde{+} \cdots \tilde{+} \lambda_{r} K_{r}=\left\{\lambda_{1} x_{1} \tilde{+} \cdots \tilde{+} \lambda_{r} x_{r}: x_{i} \in K_{i}\right\} .
$$

The following property will be used later. If $K, L \in \varphi^{n}$ and $\lambda, \mu \geq 0$

$$
\rho(\lambda K \tilde{+} \mu L, \cdot)=\lambda \rho(K, \cdot)+\mu \rho(L, \cdot) .
$$

For $K_{1}, \ldots, K_{r} \in \varphi^{n}$ and $\lambda_{1}, \ldots, \lambda_{r} \geq 0$, the volume of the radial Minkowski linear combination $\lambda_{1} K_{1} \tilde{+} \cdots \tilde{+} \lambda_{r} K_{r}$ is a homogeneous $n$ th-degree polynomial in the $\lambda_{i}$ [19],

$$
V\left(\lambda_{1} K_{1} \tilde{+} \cdots \tilde{+} \lambda_{r} K_{r}\right)=\sum \tilde{V}_{i_{1}, \ldots, i_{n}} \lambda_{i_{1}} \cdots \lambda_{i_{n}}
$$

where the sum is taken over all $n$-tuples $\left(i_{1}, \ldots, i_{n}\right)$ whose entries are positive integers not exceeding $r$. If we require the coefficients of the polynomial in (3.2) 
to be symmetric in their arguments, then they are uniquely determined. The coefficient $\tilde{V}_{i_{1}, \ldots, i_{n}}$ is nonnegative and depends only on the bodies $K_{i_{1}}, \ldots, K_{i_{n}}$. It is written as $\tilde{V}\left(K_{i_{1}}, \ldots, K_{i_{n}}\right)$ and is called the dual mixed volume of $K_{i_{1}}, \ldots, K_{i_{n}}$. If $K_{1}=\cdots=K_{n-i}=K, K_{n-i+1}=\cdots=K_{n}=L$, the dual mixed volumes is written as $\tilde{V}_{i}(K, L)$. The dual mixed volumes $\tilde{V}_{i}(K, B)$ is written as $\tilde{W}_{i}(K)$.

Dual Quermassintegral is a special case of the $p$-th dual volume:

$$
\tilde{V}_{p}(K)=\frac{1}{n} \int_{S^{n-1}} \rho(K, u)^{p} d S(u),-\infty<p<\infty .
$$

Taking $p=n-i$ in $\tilde{V}_{p}(K), \tilde{V}_{p}(K)$ changes to the well know dual Quermassintegral $\tilde{W}_{i}(K)$. Taking $p=n$ in $\tilde{V}_{p}(K), \tilde{V}_{p}(K)$ changes to the well know general volume $V_{i}(K)$.

If $K_{i} \in \varphi^{n}(i=1,2, \ldots, n-1)$, then the dual mixed volume of $K_{i} \cap E_{u}(i=$ $1,2, \ldots, n-1)$ will be denoted by $\tilde{v}\left(K_{1} \cap E_{u}, \ldots, K_{n-1} \cap E_{u}\right)$. If $K_{1}=\ldots=K_{n-1-i}$ $=K$ and $K_{n-i}=\ldots=K_{n-1}=L$, then $\tilde{v}\left(K_{1} \cap E_{u}, \ldots, K_{n-1} \cap E_{u}\right)$ is written as $\tilde{v}_{i}\left(K \cap E_{u}, L \cap E_{u}\right)$. If $L=B$, then $\tilde{v}_{i}\left(K \cap E_{u}, B \cap E_{u}\right)$ is written as $\tilde{w}_{i}\left(K \cap E_{u}\right)$.

\section{INTERSECTION BODIES}

For $K \in \varphi^{n}$, there is a unique star body $I K$ whose radial function satisfies (for $\left.u \in S^{n-1}\right)$

$$
\rho(I K, u)=v\left(K \cap E_{u}\right) .
$$

It is called the intersection bodies of $K$. From a result of Busemann, it follows that $I K$ is convex if $K$ is convex and centrally symmetric with respect to the origin. Clearly any intersection body is centered.

The volume of an intersection body is given by

$$
V(I K)=\frac{1}{n} \int_{S^{n-1}} v\left(K \cap E_{u}\right)^{n} d S(u) .
$$

The mixed intersection bodies of $K_{1}, \ldots, K_{n-1} \in \varphi^{n}$, is $I\left(K_{1}, \ldots, K_{n-1}\right)$, whose radial function is defined by

$$
\rho\left(I\left(K_{1}, \ldots, K_{n-1}\right), u\right)=\tilde{v}\left(K_{1} \cap E_{u}, \ldots, K_{n-1} \cap E_{u}\right),
$$

where $\tilde{v}$ is $(n-1)$-dimensional dual mixed volume. If $K \in \varphi^{n}$ with $\rho(K, u) \in$ $C\left(S^{n-1}\right)$, and $i \in \mathbb{R}$ is positive, the intersection body of order $i$ of $K$ is the centered star body $I_{i} K$ such that [21]

$$
\rho\left(I_{i} K\right)=\frac{1}{n-1} \int_{S^{n-1}} \rho(K, u)^{n-i-1} d S(u), \text { for } u \in S^{n-1},
$$

where $I_{i} K=I(\underbrace{K, \ldots, K}_{n-i-1}, \underbrace{B, \ldots, B}_{i})$.

If $K_{1}=\cdots=\stackrel{n-i-1}{K}=\stackrel{i}{n-i-1}=\stackrel{K}{K}, K_{n-i}=\cdots=K_{n-1}=L$, then $I\left(K_{1}, \ldots, K_{n-1}\right)$ is written as $I_{i}(K, L)$. If $L=B$, then $I_{i}(K, L)$ is written as $I_{i} K$ and is called the $i$ th 
intersection body of $K$. For $I_{0} K$ simply write $I K$. The terminology was introduced by Zhang[21].

\section{MAIN RESULTS}

Lemma A. If $K, L \in \varphi^{n},-\infty<p<\infty$ and $0<j<n-1$ then

$$
\begin{aligned}
\tilde{V}_{p}(I K) & =\frac{1}{n} \int_{S^{n-1}} v\left(K \cap E_{u}\right)^{p} d S(u), \\
\tilde{V}_{p}\left(I_{j} K\right) & =\frac{1}{n} \int_{S^{n-1}} \tilde{w}_{j}\left(K \cap E_{u}\right)^{p} d S(u), \\
\tilde{V}_{p}\left(I_{j}(K, L)\right) & =\frac{1}{n} \int_{S^{n-1}} \tilde{v}_{j}\left(K \cap E_{u}, L \cap E_{u}\right)^{p} d S(u) .
\end{aligned}
$$

To prove this we use (3.3) in conjunction with the fact (4.2).

Lemma B ([11]). If $K_{1}, \ldots, K_{n} \in \varphi^{n}$, then

$$
\tilde{V}\left(K_{1}, \ldots, K_{n}\right)^{r} \leq \prod_{j=1}^{r} \tilde{V}(\underbrace{K_{j}, \ldots, K_{j}}_{r}, K_{r+1}, \ldots, K_{n}),
$$

with equality if and only if $K_{1}, \ldots, K_{n}$ are all dilations of each other. We shall need the following trivial elementary inequality:

Lemma C. If $a, b \geq 0$ and $c, d>0$ then for $0<p<1$

$$
(a+b)^{p}(c+d)^{1-p} \geq a^{p} c^{p-1}+b^{p} d^{p-1},
$$

with equality if and only if $a d=b c$.

\section{THE MinKows KI INEQUALITY FOR DUAL QUERMASSINTEgRAL SUM OF MIXED INTERSECTION BODIES}

In [10], Leng introduced the concept of $i$-Quermassintegral difference function of convex bodies as follows. If $K, D \in \mathcal{K}^{n}$ and $D \subset K$, then the $i$ - Quermassintegral difference function of convex bodies $K$ and $D, D_{w_{i}}(K, D)$, was defined by

$$
D_{w_{i}}(K, D)=W_{i}(K)-W_{i}(D)(0 \leq i \leq n-1) .
$$

In the section, we first introduce a new concept, dual Quermassintegral sum function, as follows.

If $K, D \in \varphi^{n}$, then the $p$-dual volume sum function of star bodies $K$ and $D$, $S_{\tilde{v}_{p}}(K, D)$, is defined by

$$
S_{\tilde{v}_{p}}(K, D)=\tilde{V}_{p}(K)+\tilde{V}_{p}(D) .
$$

When $p=n$, we have $S_{v}(K, D)=V(K)+V(D)$, which is called the dual volume sum function of star bodies $K$ and $L$. 
The following Minkowski inequality for mixed intersection bodies will be established: If $K, L \in \varphi^{n}$, and $0 \leq i<n$ and $0<j<n-1$, then

$$
\tilde{W}_{i}\left(I_{j}(K, L)\right)^{n-1} \leq \tilde{W}_{i}(I K)^{n-j-1} \tilde{W}_{i}(I L)^{j},
$$

with equality if and only if $K$ and $L$ are dilates.

This is just the special $D=D^{\prime}=\varnothing$ case of:

Theorem 1. If $K, L, D, D^{\prime} \in \varphi^{n}$. Let $D^{\prime}$ is a dilates copy of $D$, and $-\infty<p<\infty$ and $0<j<n-1$, then

$$
S_{\tilde{v}_{p}}\left(I_{j}(K, L), I_{j}\left(D, D^{\prime}\right)\right)^{n-1} \leq S_{\tilde{v}_{p}}(I K, I D)^{n-j-1} S_{\tilde{v}_{p}}\left(I L, I D^{\prime}\right)^{j},
$$

with equality if and only if $K$ and $L$ are dilates.

Proof. In view of the special case of Lemma B, we obtain that

$$
\tilde{v}_{j}\left(K \cap E_{u}, L \cap E_{u}\right) \leq v\left(K \cap E_{u}\right)^{\frac{(n-j-1)}{n-1}} v\left(L \cap E_{u}\right)^{\frac{j}{n-1}} .
$$

with equality if and only if $K \cap E_{u}$ and $L \cap E_{u}$ are dilates, this happens if and only if $K$ and $L$ are dilates [20].

From Lemma A, (6.3) and in view of Minkowski inequality for integral [9], we have $j<n-1$

$$
\begin{gathered}
n \tilde{V}_{p}\left(I_{j}(K, L)\right)=\left(\left\|\tilde{v}_{j}\left(K \cap E_{u}, L \cap E_{u}\right)\right\|_{p}\right)^{p} \\
\leq\left(\left\|v\left(K \cap E_{u}\right)^{\frac{n-j-1}{n-1}} v\left(L \cap E_{u}\right)^{\frac{j}{n-1}}\right\|_{p}\right)^{p} \\
\leq\left(\| v\left(K \cap E_{u} \|_{p}\right)^{\frac{p(n-j-1)}{n-1}}\left(\| v\left(K \cap E_{u} \|_{p}\right)^{\frac{j p}{n-1}}\right.\right. \\
=\left(n \tilde{V}_{p}(I K)\right)^{\frac{(n-j-1)}{n-1}}\left(n \tilde{V}_{p}(I L)\right)^{\frac{j}{n-1}} \\
=n \tilde{V}_{p}(I K)^{\frac{(n-j-1)}{n-1}} \tilde{V}_{p}(I L)^{\frac{j}{n-1}} .
\end{gathered}
$$

In view of the conditions of (6.3) and the Minkowski inequality for the integral, it follows that the equality holds if and only if $K$ and $L$ are dilates.

Moreover, we consider the case of $j=n-1$ of the inequality (6.4). If $j=n-1$, inequality (6.4) reduces to

$$
\tilde{W}_{n-1}\left(I_{j}(K, L)\right)^{n-1} \leq \tilde{W}_{n-1}(I K)^{n-j-1} \tilde{W}_{n-1}(I L)^{j} .
$$

From Lemma A, (6.5) changes to

$$
\begin{gathered}
\left(\int_{S^{n-1}} \tilde{v}_{j}\left(K \cap E_{u}, L \cap E_{u}\right) d S(u)\right)^{n-1} \\
\leq\left(\int_{S^{n-1}} v\left(K \cap E_{u}\right) d S(u)\right)^{n-j-1}\left(\int_{S^{n-1}} v\left(L \cap E_{u}\right) d S(u)\right)^{j} .
\end{gathered}
$$


On the other hand, integrating two sides of (6.3) and in view of Hölder inequality for integral, we obtain that

$$
\begin{gathered}
\int_{S^{n-1}} \tilde{v}_{j}\left(K \cap E_{u}, L \cap E_{u}\right) d S(u) \\
\leq \int_{S^{n-1}} v\left(K \cap E_{u}\right)^{\frac{n-j-1}{n-1}} v\left(L \cap E_{u}\right)^{\frac{j}{n-1}} d S(u) \\
\leq\left(\int_{S^{n-1}} v\left(K \cap E_{u}\right) d S(u)\right)^{\frac{n-j-1}{n-1}}\left(\int_{S^{n-1}} v\left(L \cap E_{u}\right) d S(u)\right)^{\frac{j}{n-1}} .
\end{gathered}
$$

Moreover, from the inequality (6.4), we obtain that

$$
\tilde{V}_{p}\left(I_{j}(K, L)\right)^{n-1} \leq \tilde{V}_{p}(I K)^{n-j-1} \tilde{V}_{p}(I L)^{j},
$$

with equality if and only if $K$ and $L$ are dilates, and

$$
\tilde{V}_{p}\left(I_{j}\left(D, D^{\prime}\right)\right)^{n-1}=\tilde{V}_{p}(I D)^{n-j-1} \tilde{V}_{p}\left(I D^{\prime}\right)^{j} .
$$

Hence, from the inequality in Lemma $\mathrm{C}$, we have

$$
\begin{gathered}
S_{\tilde{v}_{p}}\left(I_{j}(K, L), I_{j}\left(D, D^{\prime}\right)\right) \\
\leq \tilde{V}_{p}(I K)^{(n-j-1) /(n-1)} \tilde{V}_{p}(I L)^{j /(n-1)}+\tilde{V}_{p}(I D)^{(n-j-1) /(n-1)} \tilde{V}_{p}\left(I D^{\prime}\right)^{j /(n-1)} \\
\leq S_{\tilde{v}_{p}}(I K, I D)^{n-j-1} S_{\tilde{v}_{p}}\left(I L, I D^{\prime}\right)^{j}
\end{gathered}
$$

The proof of Theorem 1 is complete.

Remark 1. Let $D$ and $D^{\prime}$ be single points and take $p=n-i$ in (6.2), then (6.2) changes to inequality (6.1).

$$
\tilde{W}_{i}\left(I_{1}(K, L)\right)^{n-1} \leq \tilde{W}_{i}(I K)^{n-2} \tilde{W}_{i}(I L),
$$

with equality if and only if $K$ and $L$ are dilates.

This is just a dual form of the following inequality which was given by Lutwak[17].

Minkowski inequality for mixed projection bodies If $K, L \in \mathcal{K}^{n}$, and $0 \leq i<n$, then

$$
W_{i}\left(\Pi_{1}(K, L)\right)^{n-1} \geq W_{i}(\Pi K)^{n-2} W_{i}(\Pi L),
$$

with equality if and only if $K$ and $L$ are homothetic.

Taking $p=n-i$ in (6.2), it changes to

$$
S_{\tilde{w}_{i}}\left(I_{j}(K, L), I_{j}\left(D, D^{\prime}\right)\right)^{n-1} \leq S_{\tilde{w}_{i}}(I K, I D)^{n-j-1} S_{\tilde{w}_{i}}\left(I L, I D^{\prime}\right)^{j},
$$

with equality if and only if $K$ and $L$ are dilates.

This is just a rederivation of a recent result of Zhao and Leng[22].

Let $D$ and $D^{\prime}$ be single points and take $p=n$ in (6.2), then it changes to the well know Minkowski inequality for mixed projection bodies. 


\section{REFERENCES}

[1] K. Ball, "Shadows of convex bodies," Trans. Amer. Math. Soc., vol. 327, no. 2, pp. 891-901, 1991.

[2] K. Ball, "Volume ratios and a reverse isoperimetric inequality," J. London Math. Soc. (2), vol. 44, no. 2, pp. 351-359, 1991.

[3] J. Bourgain, "On the busemann-petty problem for perturbations of the ball," Geom. Funct. Anal., vol. 1 , no. 1 , pp. 1-13, 1991.

[4] H. Busemann, "A theorem on convex bodies of the brunn-minkowski type," Proc. Nat. Acad. Sci. U. S. A., vol. 35, pp. 27-31, 1949.

[5] H. Busemann, "The foundations of minkowskian geometry," Comment. Math. Helv., vol. 24, pp. 156-187, 1950.

[6] R. J. Gardner, "Intersection bodies and the busemann-petty problem," Trans. Amer. Math. Soc., vol. 342, no. 1, pp. 435-445, 1994.

[7] R. J. Gardner, "A positive answer to the busemann-petty problem in three dimensions," Ann. of Math. (2), vol. 140, no. 2, pp. 435-447, 1994.

[8] R. J. Gardner, Geometric tomography, ser. Encyclopedia of Mathematics and its Applications. Cambridge: Cambridge University Press, 1995, vol. 58.

[9] G. H. Hardy, J. E. Littlewood, and G. Pólya, Inequalities. Cambridge: Cambridge University Press, 1934.

[10] G.-S. Leng, "The brunn-minkowski inequality for volume differences," Adv. in Appl. Math., vol. 32, no. 3, pp. 615-624, 2004.

[11] E. Lutwak, "Dual mixed volumes," Pacific J. Math., vol. 58, no. 2, pp. 531-538, 1975.

[12] E. Lutwak, "Mixed projection inequalities," Trans. Amer. Math. Soc., vol. 287, no. 1, pp. 91-105, 1985.

[13] E. Lutwak, "Intersection bodies and dual mixed volumes," Adv. in Math., vol. 71, no. 2, pp. 232261, 1988.

[14] E. Lutwak, "Centroid bodies and dual mixed volumes," Proc. London Math. Soc. (3), vol. 60, no. 2, pp. 365-391, 1990.

[15] E. Lutwak, "On quermassintegrals of mixed projection bodies," Geom. Dedicata, vol. 33, no. 1, pp. 51-58, 1990.

[16] E. Lutwak, "The brunn-minkowski-firey theory. i. mixed volumes and the minkowski problem," $J$. Differential Geom., vol. 38, no. 1, pp. 131-150, 1993.

[17] E. Lutwak, "Inequalities for mixed projection bodies," Trans. Amer. Math. Soc., vol. 339, no. 2, pp. 901-916, 1993.

[18] E. Lutwak, "The brunn-minkowski-firey theory. ii. affine and geominimal surface areas," Adv. Math., vol. 118, no. 2, pp. 244-294, 1996.

[19] R. Schneider, Convex bodies: the Brunn-Minkowski theory, ser. Encyclopedia of Mathematics and its Applications. Cambridge: Cambridge University Press, 1993, vol. 44.

[20] W. Süss, "Zusammensetzung von eikörpern und homothetische eiflächen," Tohoku Math. J., vol. 35, pp. 47-50, 1932.

[21] G. Y. Zhang, "Centered bodies and dual mixed volumes," Trans. Amer. Math. Soc., vol. 345, no. 2, pp. 777-801, 1994.

[22] C.-J. Zhao and G.-S. Leng, "Inequalities for dual quermassintegrals of mixed intersection bodies," Proc. Indian Acad. Sci. Math. Sci., vol. 115, no. 1, pp. 79-91, 2005. 
Authors' addresses

Zhao Chang-jian

Department of Information and Mathematics Sciences, College of Science,, China Jiliang University, Hangzhou 310018, P. R. China

E-mail address: chjzhao@yahoo.com.cn, chjzhao@163.com, chjzhao@cjlu.edu.cn

Mihály Bencze

Str. Hărmanului 6, 505600 Săcele-Négyfalu, Jud. Brasov, Romania

E-mail address: aedit@metanet.ro 